\title{
ON INVERSIONS IN NORMED LINEAR SPACES
}

\author{
W. J. STILES
}

If $E$ is a normed linear space and $S=\{x \in E:\|x\|=1\}$ is the unit sphere of $E$, the inversion of $E$ in $S$ is the mapping of $E \sim\{0\}$ onto itself which carries a point $x \neq 0$ in $E$ onto the point $x /\|x\|^{2}$. If $E$ is an inner-product space, it is well known [4] that the inversive image of a sphere not containing the origin is a sphere, and that the inversive image of the elements of the collection of all spheres and hyperplanes is again the collection of all spheres and hyperplanes if an appropriate convention is made to take care of the origin. Our intent here is to show that the converse of this is true. In particular, we will show that if the inversive image of every hyperplane has a center of symmetry or if the inversive image of some hyperplane is a sphere and $E$ is two dimensional, then $E$ is an inner-product space. In the following, we will, for notational convenience, assume that the origin is contained in the inversive image of all hyperplanes, and we will use the term convex surface to denote the boundary of a convex body.

THEOREM. If $E$ is two-dimensional and the inversive image of some hyperplane $H$ is a sphere in $E$, then $E$ is an inner-product space.

Proof. If the inversive image of $H$ is a sphere, it is easily verified that the inversive image of $H_{x}$ is also a sphere, where $H_{x}$ is a hyperplane parallel to $H$ and supporting $S$ and $x$. Construct a line through the origin parallel to $H$ and let $y$ be a point of $S$ which is on this line. Impose a Euclidean coordinate system on $E$ in such a manner that the point $x$ is denoted by $(0,1)$ and the point of $y$ by $(1,0)$. To prove the theorem, it will suffice to show that $S$ is the unit circle in $E^{2}$.

If $C$ denotes the inversive image of $H_{x}$, then, since $2 C$ is a translate of $S, C \cap S=\{(0,1)\}$; furthermore $C$ is supported at the origin by a horizontal line. These facts imply that the center of $C$ must lie on the line segment connecting the origin and the point $(0,1)$ and, therefore, must be the point $\left(0, \frac{1}{2}\right)$.

Let $z$ be any point on $S$ lying in the upper half plane $\{(x, y): y>0\}$. Construct a ray $r_{1}$ emanating from the point $\left(0, \frac{1}{2}\right)$ and parallel to the ray emanating from the origin and containing the point $z$. Let $\bar{z}_{1}$ denote the intersection of $r_{1}$ and $C$ and let $\bar{z}_{1}^{\prime}$ denote the intersection 1967.

Received by the editors September 12, 1967 and, in revised form, November 1, 
of $-r_{1}$ and $C$. Construct two rays $r_{2}$ and $r_{2}^{\prime}$ emanating from the origin and containing the points $\bar{z}_{1}$ and $\bar{z}_{1}^{\prime}$, respectively. Let $z_{1}$ be the point common to $r_{2}$ and $S$ and let $z_{1}^{\prime}$ be the point common to $r_{2}^{\prime}$ and $S$. Let $\mathcal{E}_{z}$ denote the ellipse with a vertical axis which is centered at the origin, contains the point $z$ and has the lines $y= \pm 1$ as supports. Since the inversive image of $H_{x}$ in $\mathcal{E}_{z}$ is an ellipse similar to $\mathcal{E}_{z}$ and since $\bar{z}_{1}$ lies on this ellipse, the point $z_{1}$, being uniquely determined by $\bar{z}_{1}$ must lie on $\varepsilon_{z}$ as well as on $S$. Similarly $z_{1}^{\prime}$ must lie on $\varepsilon_{z}$ as well as on $S$. Repeating the above, we can obtain two sequences, $\left\{z_{n}\right\}$ and $\left\{z_{n}^{\prime}\right\}$, such that $z_{n}$ and $z_{n}^{\prime}$ lie both on $\varepsilon_{z}$ and on $S$. The sequence $\left\{z_{n}\right\}$ must converge to the point $(0,1)$ while the sequence $\left\{z_{n}^{\prime}\right\}$ must converge to the point $(-1,0)$. The fact that the sequence $\left\{z_{n}^{\prime}\right\}$ converges to $(-1,0)$ implies that $\mathcal{E}_{z}$ must be the unit circle. Hence every point of $S$ in the upper half plane lies on the unit circle, and, by the symmetry of $S$, every point of $S$ lies on the unit circle.

It is not difficult to construct examples which show that the preceding theorem does not hold in higher dimensional spaces. In fact, if $E$ is any normed linear space whose norm is denoted by \|\|$E$ and if $R$ is the set of all real numbers, the space $R \times E$ with norm given by $\|(z, x)\|=\left(z^{2}+\|x\|^{2}\right)^{1 / 2}$ is such that the inversive image of the hyperplane $H=\{(z, x): z=1\}$ is similar to the unit sphere of $R \times E$.

Before proceeding to the proof of the final theorem we note, without proof, that the inversive image of any hyperplane is a convex surface, and if $S$ is strictly convex, the inversive image is a strictly convex surface. We also note that when the space is smooth and two dimensional, the direction of a line of support of the inversive image depends only upon the direction of the line of support of the corresponding point on $S$. This last statement can be seen as follows:

Suppose that $S$ is a symmetric convex body centered at the origin whose equation in polar coordinates is $r=f(\theta)$. Suppose further that $f(\pi / 2)=1$ and that $S$ is supported by the lines $y= \pm 1$; then the equation for the inversive image of the line $y=1$ in $S$ is easily seen to be $r=f^{2}(\theta) \sin \theta$. If $\psi(\theta), \psi_{1}(\theta)$ denote the angle that the respective support lines of $S$ and of the inversive image of $y=1$ make with a radial vector, then $\cot \psi_{1}(\theta)=2 \cot \psi(\theta)+\cot \theta$.

Finally, we note that if the inversive image of every support line in the unit sphere of a two-dimensional space is symmetric, the unit sphere must be smooth.

THEOREM. If in any normed linear space $E$ the inversive image of every supporting hyperplane has a center of symmetry, then $E$ is an inner-product space. 
Proof. Because of the well-known Jordan-von Neumann characterization of inner-product spaces [2], we need only show that every two-dimensional subspace of $E$ is an inner-product space. Let $F$ be any two-dimensional subspace of $E$ and let $S_{F}$ be the restriction of $S$ to $F$. Let $x$ be any point of $S_{F}$ and $H_{F}$ any supporting hyperplane of $S_{F}$ in $F$. If $H$ is a supporting hyperplane of $S$ in $E$ which contains $H_{F}$, then the inversive image, $C$, of $H$ in $S$ is symmetric, $C$ is supported at the origin only by a translate of $H$ and $C \cap H$ is the null vector. This implies that $S \cap H=\{x\}$ which in turn implies that the center of $C$ is the point $\frac{1}{2} x$. The above argument shows that the inversive image of every supporting hyperplane of $S_{F}$ in $F$ is symmetric about the point $\frac{1}{2} x$ where $x$ is the (unique) point of support. We will complete the proof by showing that $F$ is an inner-product space.

In [1], M. Day has shown that in any two-dimensional space there are two vectors $x$ and $y$ which are mutually orthogonal, where $x$ is said to be orthogonal to $y$ if and only if $\|x\| \leqq\|x+t y\|$ for all real numbers $t$. Let $x$ and $y$ be two mutually orthogonal unit vectors in $F$, and let $T$ denote the linear mapping of $F$ onto two-dimensional Euclidean space, $E^{2}$, which takes the point $x$ onto the point $(0,1)$ and the point $y$ onto the point $(1,0)$. Since $T$ is an isometry of $F$ onto the space whose unit ball is $T\left(S_{F}\right)$, the inversive image of every supporting hyperplane to $T\left(S_{F}\right)$ is symmetric. By the choice of $x$ and $y$ in $F$, the lines $x= \pm 1$ and $y= \pm 1$ are lines of support of $T\left(S_{F}\right)$ in $E^{2}$.

Let $z$ by any point on the unit circle other than $(1,0)$ or $(0,1)$. We will show that $z$ lies on $T\left(S_{F}\right)$. To show this, we construct the line of support, $L$, of the unit circle at the point $z$, and we observe that the inversive image, $\bar{L}$, of $L$ in $T\left(S_{F}\right)$ behaves like the inversive image of $L$ in the unit circle at the two points $(1,0)$ and $(0,1)$. In particular, if $L$ intersects the vertical and horizontal axes in the points $w_{1}$ and $w_{2}$, respectively, then the inversive image of $L$ has parallel lines of support at the points $\bar{w}_{1}$ and $\bar{w}_{2}$, where $\bar{w}_{1}$ and $\bar{w}_{2}$ are the inversive images (in $T\left(S_{F}\right)$ ) of the points $w_{1}$ and $w_{2}$. Since the inversive image of every supporting line is symmetric, $T\left(S_{F}\right)$ must be strictly convex, and since $T\left(S_{F}\right)$ is strictly convex, the inversive image of each line inverted in $T\left(S_{F}\right)$ must be strictly convex. This implies that the center of the inversive image of $L$ must be the point $\frac{1}{2}\left(\bar{w}_{1}+\bar{w}_{2}\right)$. But this point is easily seen to be $\frac{1}{2} z$. Since $\bar{L}$ is symmetric with center of symmetry at $\frac{1}{2} z, z$ must also lie on $\bar{L}$ and hence on $T\left(S_{F}\right)$. Since each point of the unit circle is a point of $T\left(S_{F}\right), T\left(S_{F}\right)$ must be the unit circle, and, since $T$ is an isometry, $F$ must be an inner product space.

We close by noting that inversions in $E^{n}$ and their relationship to convexity have been investigated by P. Kelly and E. Straus in [3]. 


\section{REFERENCES}

1. M. Day, Polygons circumscribed about closed convex curves, Trans. Amer. Math. Soc. 62 (1947), 315-319.

2. $\mathrm{P}$. Jordan and $\mathrm{J}$. von Neumann, On inner products in linear metric spaces, Ann. of Math. (2) 36 (1935), 719-723.

3. P. Kelly and E. Straus, Inversive and conformal convexity, Proc. Amer. Math. Soc. 8 (1957), 572-577.

4. V. Klee, Convexity of Chebyshev sets, Math. Ann. 142 (1961), 292-304.

Florida State University 